\title{
Blepharospasm-oromandibular dystonia syndrome
}

INSERM

\section{Source}

INSERM. (1999). Orphanet: an online rare disease and orphan drug data base.

Blepharospasm-oromandibular dystonia syndrome. ORPHA:93964

Blepharospasm-oromandibular dystonia, also called Meige dystonia or Meige syndrome

is a focal dystonia (see this term) involving symmetrical benign essential blepharospasm (BEB) and oromandibular dystonia (see these terms). 